\title{
A mutation in a CD44 variant of inflammatory cells enhances the mitogenic interaction of FGF with its receptor
}

\author{
Shlomo Nedvetzki, ${ }^{1}$ Itshak Golan, ${ }^{1}$ Nathalie Assayag, ${ }^{1}$ Erez Gonen, ${ }^{1}$ Dan Caspi, ${ }^{2}$ \\ Micha Gladnikoff, ${ }^{3}$ Avner Yayon, ${ }^{3}$ and David Naor ${ }^{1}$ \\ ${ }^{1}$ The Lautenberg Center for General and Tumor Immunology, The Hebrew University - Hadassah Medical School,
Jerusalem, Israel
${ }^{2}$ Tel Aviv Souraski Medical Center, Tel Aviv University, Department of Rheumatology, Tel Aviv, Israel
${ }^{3}$ ProChon Biotech Ltd., Kiryat Weizmann, Science Park, Rehovot, Israel
}

\begin{abstract}
Synovial fluid cells from joints of rheumatoid arthritis (RA) patients express a novel variant of CD44 (designated CD44vRA), encoding an extra trinucleotide (CAG) transcribed from intronic sequences flanking a variant exon. The CD44vRA mutant was detected in 23 out of 30 RA patients. CD44-negative Namalwa cells transfected with CD44vRA cDNA or with CD44v3-v10 (CD44vRA wild type) cDNA bound FGF-2 to an equal extent via their associated heparan sulfate chains. However, Namalwa cells, immobilizing FGF-2 via their cell surface CD44vRA, bound substantially more soluble FGF receptor-1 (FGFR-1) than did Namalwa cells immobilizing the same amount of FGF-2 via their cell surface CD44v3-v10. The former cells stimulated the proliferation of BaF-32 cells, bearing FGFR-1, more efficiently than did the latter cells. Finally, isolated primary synovial fluid cells from RA patients expressing CD44vRA bound more soluble FGFR-1 to their cell surface-associated FGF-2 than did corresponding synovial cells expressing CD44v3-v10 or synovial cells from osteoarthritis patients. The binding of soluble FGFR-1 to RA synovial cells could be specifically reduced by their preincubation with Ab's against the v3 exon product of CD44. Hence, FGF-2 attached to the heparan sulfate moiety expressed by the novel CD44 variant of RA synovium cells exhibits an augmented ability to stimulate FGFR-1-mediated activities. A similar mechanism may foster the destructive inflammatory cascade not only in RA, but also in other autoimmune diseases.
\end{abstract}

J. Clin. Invest. 111:1211-1220 (2003). doi:10.1172/JCI200317100.

\section{Introduction}

CD44 is a cell surface glycoprotein involved in multiple cellular functions, including cell matrix interactions, cell migration, programmed cell death (apoptosis), or, conversely, cell survival and proliferation. Additionally, CD44 isoforms were shown to exert some of their functions through docking and presentation of cytokines, chemokines, growth factors, and enzymes to their relevant cell surface receptors or substrates $(1,2)$. Hyaluronic acid is the principal ligand of CD44 (3), but other cell

\section{Received for publication October 10, 2002, and accepted in revised form} February 11, 2003.

Address correspondence to: David Naor, The Lautenberg Center for General and Tumor Immunology, The Hebrew University Hadassah Medical School, Jerusalem 91120, Israel.

Phone: 972-2-675-8722; Fax: 972-2-642-4653;

E-mail: naord@md2.huji.ac.il.

Shlomo Nedvetzki and Itshak Golan contributed equally to this work.

Conflict of interest: The authors have declared that no conflict of interest exists.

Nonstandard abbreviations used: CD44 variant (CD44v); standard CD44 (CD44s); glycosaminoglycan (GAG); heparan sulfate (HS); rheumatoid arthritis (RA); PBS containing $0.1 \%$ Tween-20 (PBS-T); recombinant human FGF-2 (rhFGF-2); phycoerythrin (PE); FGF receptor-1 (FGFR-1); alkaline phosphatase (AP); osteoarthritis (OA); serine-glycine (SG); adjuvant-induced arthritis (AIA). surface or ECM components, such as osteopontin, fibrinogen, fibronectin, collagen, and laminin, can interact with this glycoprotein, as well (1). This multifunctionality of CD44 is possible due to the tremendous structural variability of this receptor, derived from its highly complex genetic construction. Theoretically, hundreds of CD44 isoforms can be generated by alternative splicing (4) of ten (mouse) or nine (human) variant exons, designated $\mathrm{v} 1$ to $\mathrm{v} 10$, inserted in different combinations between the two constant regions consisting of five exons at one end of the molecule and four at the other (1-3). The number of CD44 variants (CD44v) identified so far, however, is limited to a few dozen, detected mostly on epithelial cells, keratinocytes, activated leukocytes, and many types of tumor cells (2). Direct splicing of constant exon 5 to constant exon 16 (thereby skipping all the variant exons) generates the standard CD44 (CD44s), ubiquitously expressed on mesenchymal cells and on all types of hematopoietic cells (1-3). While alternative splicing is a most efficient means of enriching the genetic information stored in a single gene, posttranslational modifications by glycosylation and glycosaminoglycan (GAG) attachments further modify the CD44 protein, allowing greater expansion of its variability and functions (1-3). Indeed, it has been found that heparan sulfate (HS) attached to the v3 exon of v3-containing 
CD44 proteoglycans can immobilize HS-binding growth factors, such as hepatocyte growth factor/scatter factor (5-7), VEGF (8), and heparin-binding epidermal growth factor (8-10), as well as FGF-2 (also known as basic FGF) (8, 9, 11), FGF-4 (12), and FGF-8 (12). Previous studies on the mode of action of FGF-2 and its association with HS identified a novel role for cell surface- and ECM-resident HS molecules in the formation of distinct FGF-2-HS complexes that are essential for binding FGF-2 to its cognate receptor (13) and subsequent signal transduction $(14,15)$. The crucial role of cell surface HS was revealed by the finding that high-affinity receptor binding of FGF-2 is abolished in $\mathrm{CHO}$ mutant cell lines defective in the metabolism of GAGs (13). Receptor binding was fully restored upon addition of exogenous heparin $(13,15)$. Subsequent analysis of the conditions required for high-affinity binding of FGF-2, using soluble recombinant receptors, confirmed the importance of the initial formation of specific FGF-2-HS-receptor complexes for highaffinity binding and activation of FGF receptors (15). The growth factor-binding function of $\mathrm{v} 3$-containing CD44 can support both physiological (e.g., embryonic limb outgrowth) (12) and pathological (e.g., tumor cell motility and growth) $(6,16)$ activities. We (17-19) and others (20-23) have shown that CD44 targeting by anti-CD44 mAb's can reduce an experimental tumor growth as well as pathological activities in experimental autoimmune diseases, possibly by disrupting CD44-dependent functions, such as cell migration (24). In most cases, the mAb's were directed against standard CD44 epitopes, shared by all CD44 isoforms (designated pan-CD44), resulting in targeting of cells engaged in physiological and pathological activities. Therefore, we have focused new efforts on identifying disease-specific CD44 isoforms. Screening for exclusive CD44 variant sequences in synovial fluid cells derived from rheumatoid arthritis (RA) patients, we discovered a novel isoform in which an extra intronderived trinucleotide is inserted into the CD44v3-v10 variant mRNA of the inflamed joint cells. The expressed RA-associated CD44 variant (designated CD44vRA) bound FGF-2 in a manner promoting highaffinity binding of soluble FGF receptor- 1 as well as the activation of this cell surface receptor.

\section{Methods}

Cloning and transfection of human CD44vRA, CD44v3-10, and CD44s. For cloning of human CD44vRA cDNA, the total synovial fluid cell population of RA patients undergoing joint aspiration was isolated. RNA was isolated with a commercial kit (Promega Corp., Madison, Wisconsin, USA). CD44vRA cDNA was prepared by RT-PCR (PTC-100 Programmable Thermal Controller; MJ Research, Watertown, Massachusetts, USA), using primers representing the constant coding regions of CD44 (see Figure 1): Ex1-sense, 5'-GAATTCGCCGCCACCATGGACAAGTTTTGGTGG-3'; Ex20-antisense, 5' -TCTAGATTACACCCCAATCTTCATG-3'. PCR product size was confirmed by agarose gel electrophoresis, sequencing (ABI PRISM 310; PerkinElmer Inc., Wellesley, Massachusetts, USA), and PstI (New England Biolabs Inc., Beverly, Massachusetts, USA) digestion (the nucleotide insertion in CD44vRA introduces a PstI digestion site). The PCR product was excised from the gel, purified, and subcloned into the PGEM vector (Promega Corp.). Positive clones were selected by white/blue screening. Plasmids were purified with a commercial kit (Promega Corp.) subjected to EcoRI/XbaI (New England Biolabs Inc.) double digestion and cloned into the pcDNA3.1 vector (Invitrogen Ltd., Paisley, United Kingdom) in which the gene product was expressed. The plasmid was transfected into the CD44-negative Namalwa Burkitt lymphoma cell line (American Type Culture Collection, Manassas, Virginia, USA) as described (25). For cloning of human CD44v3-10, RNA was isolated from human keratinocytes (a gift from H. Ben-Bassat, Hadassah University Hospital, Jerusalem, Israel); for cloning of human CD44s, RNA was isolated from the HeLa cervical cancer cell line (obtained from American Type Culture Collection), using the above described protocol. Transfection of CD44v3-v10 and CD44s cDNAs as well as of the pcDNA3.1 vector was performed as described above. Accordingly, the transfected Namalwa cells were designated Namalwa-CD44vRA, Namalwa-CD44v3-v10, Namalwa-CD44s, and Namalwa-Neo (Namalwa cells transfected with empty vector).

Flow cytometry. For flow cytometry, $10^{6}$ cells were incubated with $3 \mathrm{G} 5$ anti-CD44v3 mAb (IgG2b, R\&D Systems Inc., Minneapolis, Minnesota, USA), VFF7 anti-CD44v6 mAb (IgG-1; Bender MedSystem GmbH, Vienna, Austria), F-10-44-2 anti-pan-CD44 (known also as anti-CD44s) mAb (IgG-2b; Serotec Ltd., Oxford, United Kingdom), or polyclonal anti-FGF-2 Ab (Serotec Inc.) for 45 min on ice. After extensive washing, the cells were incubated with FITC-conjugated secondary anti-Ig Ab (Jackson ImmunoResearch Laboratories Inc., West Grove, Pennsylvania, USA) for $30 \mathrm{~min}$ on ice. The cells were then washed and analyzed with a Flow Cytometer (Becton Dickinson Immunocytometry Systems, San Jose, California, USA).

Western blot analysis. Cells were lysed in NP-40 buffer and $100 \mu \mathrm{g}$ of protein, run on denaturing SDS-PAGE, and transferred to a PVDF membrane (Millipore Corp., Bedford, Massachusetts, USA). Blots were blocked with 1\% BSA in PBS containing 0.1\% Tween-20 (PBS-T) and incubated for $1 \mathrm{~h}$ with $1 \mu \mathrm{g} / \mathrm{ml}$ polyclonal anti-FGF-2 $\mathrm{Ab}$ or $2 \mu \mathrm{g} / \mathrm{ml}$ Hermes-3 anti-pan-CD $44 \mathrm{mAb}$, which was obtained from American Type Culture Collection hybridoma supernatant and purified on a protein-G column. The blots were rewashed in PBS-T, incubated with the appropriate HRP-conjugated anti-Ig secondary $\mathrm{Ab}$ (1:10,000 dilution; Jackson ImmunoResearch Laboratories Inc.) for $45 \mathrm{~min}$, rewashed in PBS-T, and developed with ECL reagent (Amersham Biosciences Ltd., Little Chalfont, United Kingdom).

FGF-2-binding assay. Biotinylated FGF-2 was prepared as follows: $100 \mu \mathrm{g}$ of recombinant human FGF-2 
(rhFGF-2) (R\&D Systems Inc.) was mixed with $13.44 \mu \mathrm{g}$ NHS-LC-Biotin (Pierce Chemical Co., Rockford, Illinois, USA) in 5\% DMSO and $0.1 \mathrm{M}$ bicarbonate buffer in a total volume of $200 \mu \mathrm{l}$. The mixture was incubated for $1 \mathrm{~h}$ at $37^{\circ} \mathrm{C}$ and stored at $4^{\circ} \mathrm{C}$. A quantity of $10^{6}$ Namalwa transfectants were incubated with $10 \mathrm{nM}$ biotinylated FGF-2 for 45 min on ice, washed once with or without $0.2 \mathrm{M} \mathrm{NaCl}$ and twice with PBS, incubated with streptavidin-phycoerythrin (streptavidin-PE) (Jackson ImmunoResearch Laboratories Inc.) for 30 min on ice, and washed and analyzed by flow cytometry. To block the binding of the FGF-2, cells were incubated with $20 \mu \mathrm{g} / \mathrm{ml}$ heparin or chondroitin sulfate A and C (Sigma Chemical Co., St. Louis, Missouri, USA), together with $10 \mathrm{nM}$ biotinylated FGF-2 for 45 min on ice and washed before FGF-2 binding analysis with streptavidin-PE, as described above. For enzymatic treatment, the cells were incubated with $20 \mathrm{mU} / \mathrm{ml}$ heparinase I or with $100 \mathrm{mU} / \mathrm{ml}$ chondroitinase $\mathrm{ABC}$ (Sigma Chemical Co.) for $2 \mathrm{~h}$ at $37^{\circ} \mathrm{C}$, washed, incubated with $10 \mathrm{nM}$ biotinylated FGF-2 for $45 \mathrm{~min}$ on ice, and rewashed before FGF-2 binding analysis with streptavidin-PE, as described above. To determine the incorporation of FGF-2 into Namalwa transfectants by Western blot analysis, the cells were incubated with 50 $\mathrm{nM}$ rhFGF-2 for $45 \mathrm{~min}$ on ice, washed with $0.2 \mathrm{M}$ $\mathrm{NaCl}$, and then subjected to SDS-PAGE and blotting with anti-FGF-2 Ab as described (12).

FGF receptor- 1 binding assay. A quantity of $5 \times 10^{6}$ Namalwa transfectants were washed with PBS and incubated with $0.1,1,2,5$, and $10 \mathrm{nM}$ rhFGF-2 for $1 \mathrm{~h}$ on ice. The cells were then washed with PBS, mixed with $200 \mu \mathrm{l}$ conditioned medium containing the soluble FGF receptor-1 (FGFR-1) ectodomain coupled to alkaline phosphatase (FGFR-1-AP) (0.24 OD units/min) (15) for $4 \mathrm{~h}$ on ice followed by extensive washing with PBS. AP substrate (pNPP solution; Sigma Chemical Co.) was added to the cells for $3 \mathrm{~h}$ at $37^{\circ} \mathrm{C}$, and the developed color was analyzed in a spectrophotometer plate reader (Molecular Devices, Sunnyvale, California, USA) at 405 $\mathrm{nm}$. To compare FGFR-1 binding with FGF-2 binding, $10^{6}$ Namalwa transfectants were washed with PBS and incubated with $10 \mathrm{nM}$ biotinylated FGF-2 for $45 \mathrm{~min}$ on ice, washed, and reincubated with streptavidin-AP (Jackson ImmunoResearch Laboratories Inc.) for $30 \mathrm{~min}$ on ice, and then rewashed extensively with PBS. The pNPP solution was added to the cells for $1 \mathrm{~h}$ at $37^{\circ} \mathrm{C}$, and the developed color was analyzed in a spectrophotometer plate reader at $405 \mathrm{~nm}$. In addition, $5 \times 10^{6}$ synovial fluid cells from the joints of RA or osteoarthritis (OA) patients, as well as primary human keratinocytes, were washed with PBS and incubated in the absence or presence of $10 \mathrm{nM}$ rhFGF-2 for $1 \mathrm{~h}$ on ice. Binding of soluble FGFR-1 to these cells was determined as described above. For blocking FGFR-1 binding, 0.01, 0.3, 3, 10 $\mu \mathrm{g} / \mathrm{ml}$ anti-CD $44 \mathrm{v} 3 \mathrm{mAb}, 10 \mu \mathrm{g} / \mathrm{ml}$ anti-pan-CD44 (F-10-44-2), or $10 \mu \mathrm{g} / \mathrm{ml}$ isotype-matched control IGg-2b (Serotec Ltd.) were added to the cells for $1 \mathrm{~h}$ on ice. After washing, the binding of FGFR-1 to these cells was determined as described above.

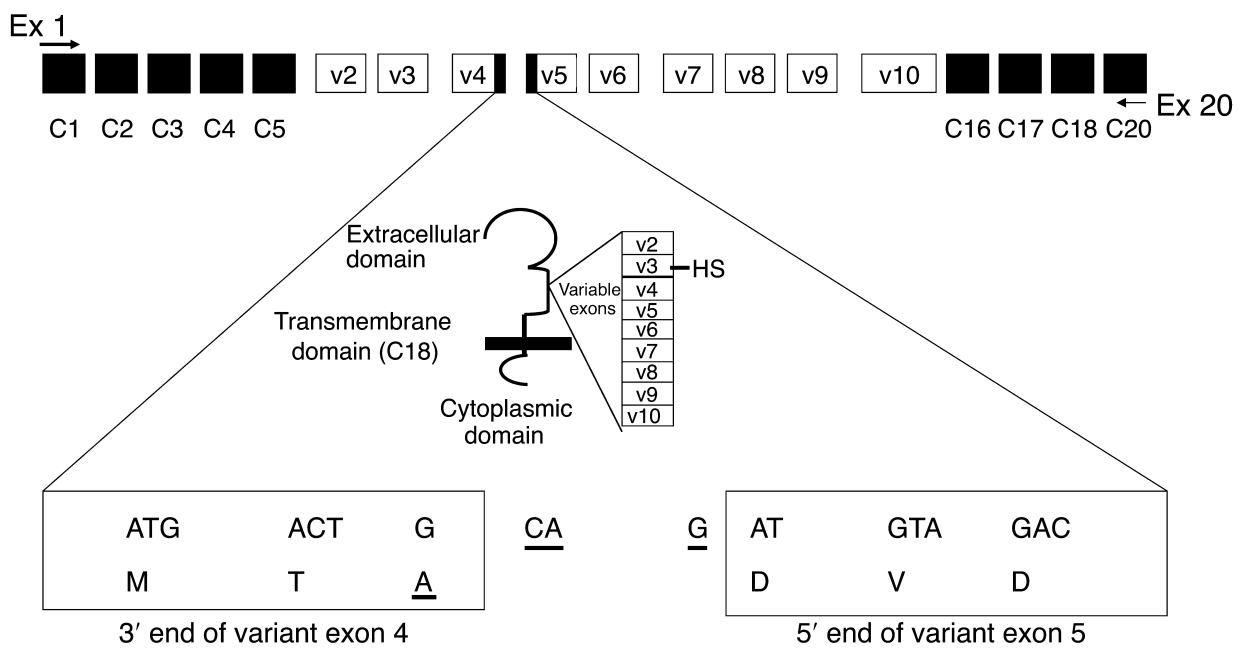

\section{Figure 1}

Schematic diagram of the CD44 molecule and the trinculeotide CAG insertion in the CD44v3-v10 sequence of RA patients. A schematic of the CD44 genomic map is shown at the top of the figure. The black squares represent the constant exons (designated C1, C2, etc.) at the two ends of the molecule. The white squares represent the variant exons (designated v2, v3, etc.) subjected to alternative splicing. Differential use of the variant exons generates the different CD44 isoforms, e.g., use of exons v3 to v10 in tandem forms the CD44v3-v10. Note that exon $v 1$ is not included in human CD44. The gap between exon v4 and exon v5 indicates the insertion site of CAG, the extra trinucleotide detected in the CD44 variant of synovial fluid cells (CD44vRA), isolated using RT-PCR from cells removed from the joints of RA patients. Arrows mark the positions and directions of Ex1 sense and Ex20 antisense primers (for sequence, see Methods) used in the above-mentioned RT-PCR. Magnification of the CAG insertion site is shown at the bottom of the figure, indicating the nucleotide sequence at the $3^{\prime}$ end of exon $v 4$ and the $5^{\prime}$ end of exon $v 5$ and its alignment with the published sequence (shown in the boxes) in the same region (29). The drawing in the middle of the figure illustrates the single chain of CD44 proteoglycan, emphasizing the position of the variable region. The HS associated with exon v3 marks the attachment site of a heparan sulfate moiety, the GAG chain that binds to FGF-2 in the CD44 variant. 
a
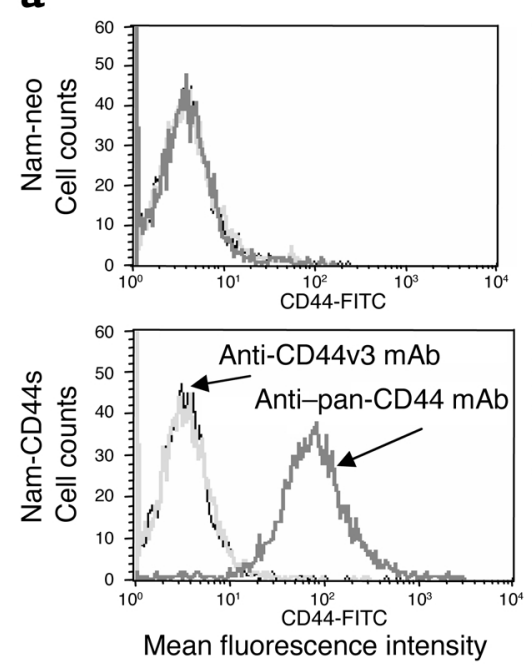
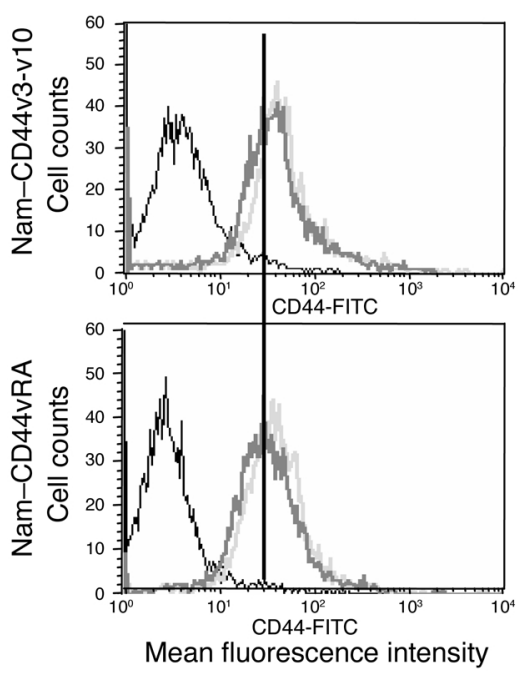

b

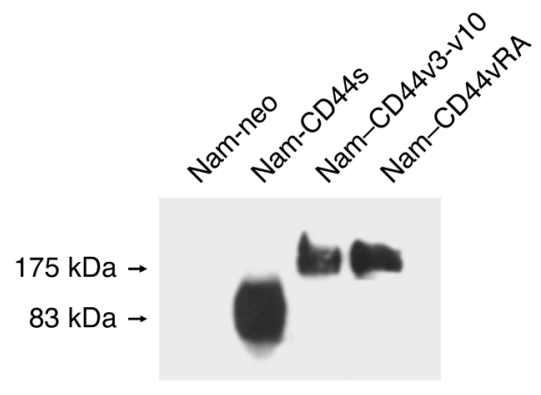

Figure 2

Binding of anti-CD44 mAbs to CD44-Namalwa (Nam) transfectants. (a) Flow cytometry. The ability of the Namalwa transfectants to interact with anti-pan CD44 mAb (F-10-44-2; dark gray line) and anti-CD44v3 mAb (light gray line) was analyzed by flow cytometry, using fluorescein-labeled anti-mouse Ig (thin black line, when the Ab was used alone) to detect the binding of the Ab's to cell surface CD44 epitopes. neo, vector including neomycin only. (b) Western blot. Western blot analysis of cell extracts from Namalwa transfectants with Hermes-3 mAb confirmed the flow-cytometry findings. Using anti-CD44 mAb, virtually equal expression of the CD44 variant exon product on Namalwa-CD44v3-v10 (11,000 densitometric units) and Namalwa-CD44vRA (11,380 units) cells and higher expression of the standard CD44 exon product on Namalwa-CD44s cells were detected.

BaF-32 cell proliferation assay. Namalwa transfectants or synovial fluid cells from the joints of RA patients were fixed with $1 \%$ paraformaldehyde (Electron Microscopy Sciences, Washington, Pennsylvania, USA) for $2 \mathrm{~h}$ on ice, washed with PBS, and placed on ice for $30 \mathrm{~min}$, rewashed three times, and suspended in RPMI-1640 containing $0.5 \%$ FCS. In a modified proliferation assay described $(26,27)$, the fixed cells were mixed with FGFR-1-transfected BaF-32 cells (a gift of Israel Vlodavsky, Hadassah University Hospital) and $1 \mathrm{nM}$ rhFGF-2. Heparin and rhFGF-2, added to BaF-32 cells as described (8), served as a positive control. BaF-32 proliferation was measured as mitochondrial NADH/NADPH-dependent dehydrogenase activity in a colorimetric assay after 72 hours, using CellTiter $96 \mathrm{AQ}_{\text {ueous }}$ One Solution Reagent (Promega Corp.), and the developed color was analyzed in a spectrophotometer plate reader at $490 \mathrm{~nm}$. To inhibit the proliferation of BaF-32 cells stimulated with Namalwa transfectants, $10 \mu \mathrm{g} / \mathrm{ml}$ of $3 \mathrm{G} 5$ anti-CD44v3 $\mathrm{mAb}$ or isotype-matched control were added to the cell mixture every 24 hours for 3 days.

Statistical analysis. Data were analyzed using microcomputer programs for one-way ANOVA, followed by the Student $t$ test for unpaired values. $P$ values less than 0.05 were considered significant. The results are expressed as the mean plus or minus SEM. Each experiment was repeated at least three times, all showing similar results.

\section{Results}

$R A s$-specific CD44 variant. In a search for disease-specific CD44 isoforms in the synovial fluid cells (comprising approximately $70 \%$ polymorphonuclears and macro- phages; the remainder are $\mathrm{T}$ and $\mathrm{B}$ lymphocytes) of RA patients, RT-PCR revealed CD44 variant transcripts, mostly CD44v3-v10, in 44 out of 47 RA patients. The expression of CD44 variants in all synovial fluid cell subpopulations was confirmed by flow cytometry (data not shown). CD44v3-v10 was also identified in normal keratinocytes (28). When the CD44v3-v10 isoform of RA synoviocytes was sequenced, we discovered that it included an extra trinucleotide (CAG) that was illegitimately transcribed from the end of the intron flanking the $5^{\prime}$ end of exon v5, allowing an extra alanine to be encoded, without interfering with the reading frame (Figure 1). Transcripts with an identical sequence change, designated CD44vRA (CD44 variant of RA patients) were detected in 23 out of $30 \mathrm{RA}$ patients whose RT-PCR products were sequenced (not shown). The inclusion of CAG, which introduced a PstI digestion site, was confirmed by PstI digestion. Preliminary evaluation using CD44vRA-specific mAb suggests that the cells expressing the novel variant constitute a major fraction of the RA synovial fluid cells, most likely macrophages and polymorphonuclear cells (data not shown). Aside from the CAG inclusion in the RA CD44 variant, CD44vRA and CD44v3-v10 have an identical sequence, shown by alignment with the entire published CD44 sequence (29), as well as with the CD44 sequence isolated from keratinocytes in our laboratory.

CD44 variants bind FGF-2 to the same extent. Namalwa cells (derived from patients with Burkitt lymphoma), originally lacking CD44, were transfected with (a) standard CD44 cDNA isolated from the HeLa cell line (designated Namalwa-CD44s), (b) CD44v3-v10 cDNA 
isolated from primary keratinocytes (NamalwaCD44v3-v10 or Namalwa-CD44v), (c) CD44v3-v10 cDNA containing the extra CAG, isolated from RA synoviocytes (Namalwa-CD44vRA), or (d) empty vector (Namalwa-Neo). Anti-pan-CD44 mAb (F-10-44-2) stained Namalwa-CD44s more intensively than Namalwa-v3-v10 and Namalwa-CD44vRA cells, but did not stain Namalwa-Neo cells. On the other hand, antiCD44v3 mAb similarly stained Namalwa-CD44v3-v10 and Namalwa-CD44vRA cells, but did not stain Namalwa-CD44s or Namalwa-Neo cells (Figure 2a). These results were confirmed by Western blot analysis with Hermes-3 anti-CD44 mAb (Figure 2b), which showed that extracts of Namalwa-CD44s include only CD44s, whereas extracts of Namalwa-CD44v3-v10, as well as of Namalwa-CD44vRA, contain only the CD44 variant. Namalwa-CD44v3-v10 and Namalwa-CD44vRA cells contain a similar amount of CD44 protein, whereas Namalwa-CD44s cells have a higher level of the ectopically expressed protein.

Heparin-binding growth factors such as FGF-2 can be bound to the V3associated $\mathrm{HS}$ of $\mathrm{v} 3$-containing CD44 variants (but not to other variant exons) and then presented autocrinally or paracrinally to the corresponding receptors (30). Flow cytometry revealed that PE-labeled FGF-2 bound at a wide range of concentrations (0.1-20 $\mathrm{nM}$ ) (not shown) at an extent similar to Namalwa-CD44v3-v10 cells and to Namalwa-CD44vRA cells (Figure 3; 10 nM FGF-2), both in the absence (Figure $3 \mathrm{a}$, inset) and in the presence (Figures 3 , a and b) of $0.2 \mathrm{M} \mathrm{NaCl}$, which reduces the nonspecific binding of FGF-2. In contrast, Namalwa-CD44s cells and Namalwa-Neo cells did not bind FGF-2 at all of the above-indicated concentrations (Figure 3, a and b; $10 \mathrm{nM}$ FGF-2). The similar binding of FGF-2 to Namalwa-CD44v3-v10 and Namalwa-CD44vRA was confirmed by Western blot analysis with antiFGF-2 Ab, using cell extracts from Namalwa transfectants expressing the corresponding CD44 variants (Figure $3 \mathrm{~b})$. The binding of PE-labeled FGF-2 to Namalwa-CD44vRA (Figure 3c) and to three of its clones (not shown), was blocked by an excess of soluble heparin, and much less so by chondroitin sulfate, indicating that the HS moiety mediates the interaction between FGF-2 and NamalwaCD44vRA. Similarly, the binding of PE-labeled FGF-2 to NamalwaCD44vRA (Figure 3d) and to three of its clones (not shown) was inhibited by pretreatment of the cells with heparinase, but not with chondroitinase ABC (Figure 3d), demonstrating that it is the HS moiety of CD44 that mediates the interaction between FGF-2 and Namalwa-CD44vRA.

FGF-2 bound to cell surface CD44vRA interacts with soluble FGFR-1 and enhances FGFR-1-mediated cell proliferation. A soluble FGFR-1 extracellular domain fused to AP showed significantly better binding to NamalwaCD44vRA cells preincubated with $5 \mathrm{nM}$ FGF-2, than to similarly treated Namalwa-CD44v3-v10 cells (Figure 4a). For comparison, we also assessed the binding of biotinylated FGF-2 to the same transfectants and found that Namalwa-CD44vRA and Namalwa-v3-v10 bound this growth factor to an equal extent (Figure 4a), confirming the results described in Figure 3 , $a$ and $b$. This finding shows that the FGF-2-mediated binding of FGFR-1 to Namalwa-CD44vRA cells is more efficient than that to Namalwa CD44v3-v10 cells, although both transfected cell lines bind similar levels
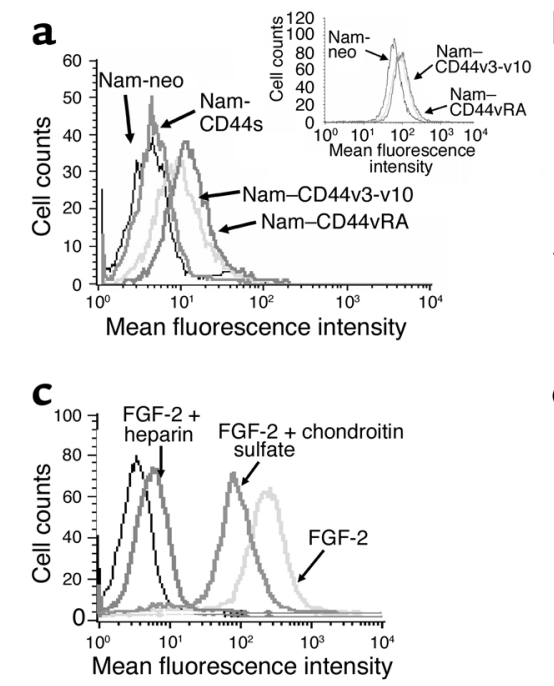

Figure 3

Namalwa-CD44v3-v10 and Namalwa-CD44vRA bind FGF-2 to a similar extent. (a) Flow cytometry. The indicated Namalwa transfectants were incubated with biotinylated FGF-2 in the absence (inset) or presence of $0.2 \mathrm{M} \mathrm{NaCl}$ and then analyzed by flow cytometry for their ability to bind this growth factor, detected by staining with streptavidin-PE. Control: Namalwa cells transfected with empty vector (Namalwa-neo) and incubated with biotinylated FGF-2. (b) Western blot analysis. Western blots of cell extracts from Namalwa transfectants with anti-FGF Ab confirmed the flow-cytometry analysis. The Namalwa transfectants were preincubated with FGF-2 before being subjected to cell extraction and gel electrophoresis. The anti-FGF Ab showed that FGF-2 was bound to a similar extent to CD44v3-v10 and CD44vRA, whereas CD44s did not bind FGF-2. Actin, a housekeeping gene product, is equally expressed in all transfectant extracts. (c) Excess soluble heparin blocks the binding of FGF-2 to NamalwaCD44vRA. Namalwa-CD44vRA cells were coincubated with biotinylated FGF-2 and an excess of soluble heparin or soluble chondroitin sulfate A plus $C$, then analyzed by flow cytometry for their ability to bind the growth factor. The binding of biotinylated FGF-2 was detected with streptavidin-PE. The extreme left-hand histogram depicts Namalwa-CD44vRA cells incubated with streptavidin-PE only. Similar results were obtained using three Namalwa-CD44vRA clones (not shown). (d) Heparinase treatment reduces FGF-2 binding to Namalwa-CD44vRA. Namalwa-CD44vRA cells were treated with heparinase or chondroitinase $A B C$ and then analyzed by flow cytometry for their ability to bind biotinylated FGF-2. Detection and control as in c. Similar results were observed in three Namalwa-CD44vRA clones (not shown). 
a
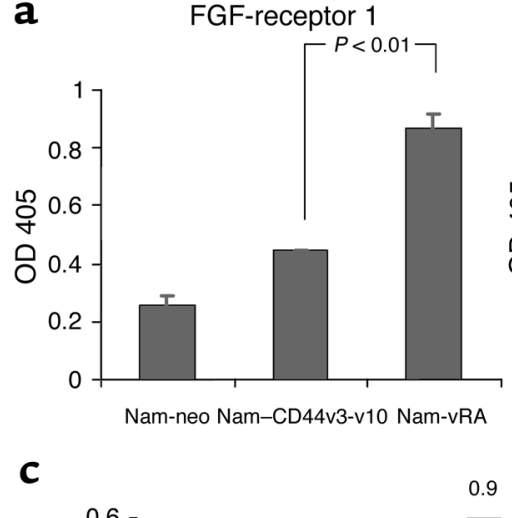

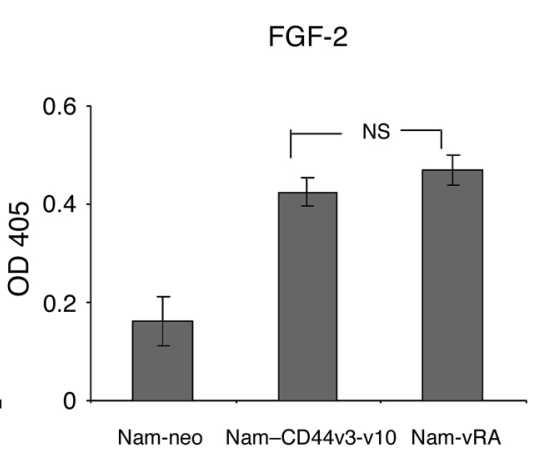

b
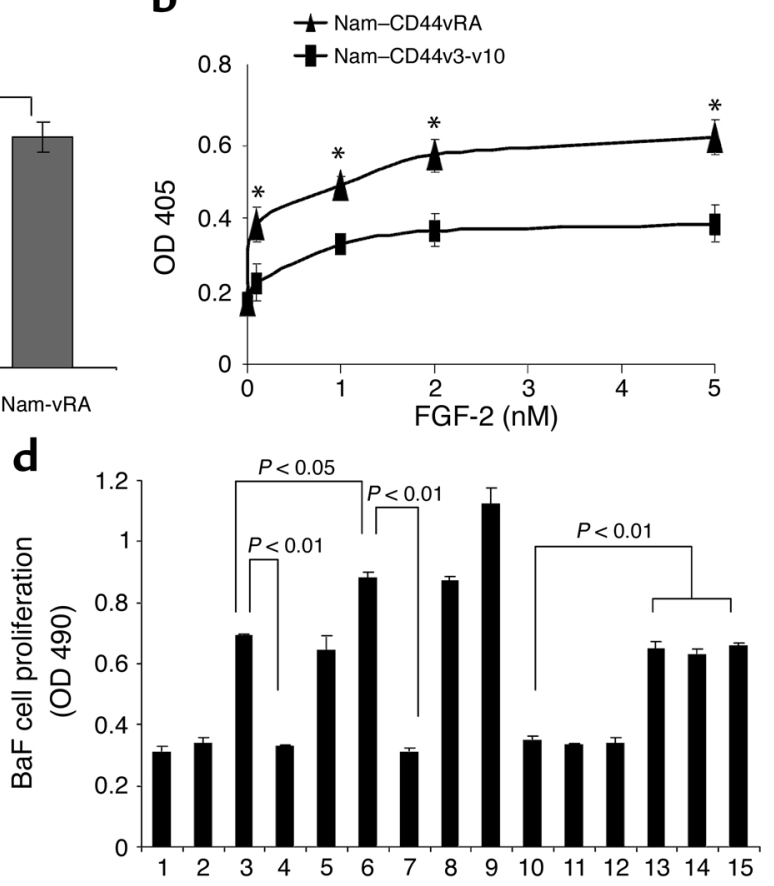

\section{Figure 4}

Enhanced binding of FGFR-1 to cell surface CD44vRA. (a) Binding of FGFR-1 to Namalwa transfectants. The ability of the indicated cells to bind soluble FGFR-1 or FGF-2 was analyzed as described in Methods. (b) Kinetics of FGFR-1 binding to FGF-2-associated Namalwa transfectants. Namalwa-CD44v3-v10 and Namalwa-CD44vRA were incubated in the presence of the indicated concentrations ofFGF-2 with soluble FGFR-1 conjugated to AP. Binding of the soluble receptor to Namalwa transfectants was assessed as indicated in a and demonstrates $50 \%$ effective binding at 100 pM of FGF-2. ${ }^{*} P<0.05$. (c) FGF-2 bound to Namalwa-CD44vRA induces enhanced proliferation of BaF-32 cells expressing FGFR-1. The indicated fixed Namalwa transfectants were incubated in the presence of FGF-2 with BaF-32 cells. The ability of the bound FGF-2 to induce proliferation in BaF-32 cells was analyzed by a colorimetric assay at OD 490 . Positive control: BaF-32 cells incubated with FGF-2 and heparin. Negative controls: BaF-32 cells incubated with FGF-2 alone or with heparin alone. Inset: A similar experiment, except that the proliferation of the positive-control BaF-32 cells is identical to the proliferation of BaF-32 cells incubated with Namalwa-CD44vRA cells. The numbers beneath the bars correspond to the numbered treatments shown under the bar of the main figure (Figure 4c). (d) Analysis of BaF-32 cell proliferation induced by fixed Namalwa transfectants or RA synovial fluid cells. Inhibition of BaF-32 cell proliferation by anti-CD44v3 mAb. The intensity of BaF-32 cell proliferation, as indicated by a colorimetric assay, following incubation with: bar 1, Namalwa-Neo cells; bar 2, Namalwa-CD44s cells; bar 3, Namalwa-CD44v3-v10 cells; bar 4, Namalwa-CD44v3-v10 cells plus antiCD44v3 mAb; bar 5, Namalwa-CD44v3-v10 cells plus isotype-matched control mAb: bar 6, Namalwa-CD44vRA cells; bar 7, NamalwaCD44vRA cells plus anti-CD44v3 mAb; bar 8, Namalwa-CD44vRA cells plus isotype-matched control mAb; bar 9, FGF-2 plus heparin (positive control); bar 10, FGF-2 alone (negative control); bar 11, heparin alone (negative control); bar 12, medium alone (negative control); bars 13-15, proliferation of BaF-32 cells following incubation with fixed RA synovial fluid cells derived from three different patients (RA16, RA17, RA18). Note that during the proliferation assay, Namalwa transfectants were loaded with FGF-2, whereas synovial fluid cells were not, because they contain endogenous FGF-2 (see inset, Figure 5b). Statistical analysis of the principal groups is shown.

of FGF-2. Repeating the direct binding experiment, this time using several different concentrations of FGF-2, gave us an opportunity to learn also about the saturation binding kinetics of this reaction (Figure $4 \mathrm{~b}$ ). From the initial rates of binding under conditions where the HP-ligand binding sites for FGF-2 are saturated and show a typically high affinity and specific binding profile, we could estimate an apparent disassociation constant of about $100 \mathrm{pM}$ for both CD44vRA- and CD44v3-v10-associated FGF-2. This is well within the known range for FGF-2 binding to cells expressing FGFR-1. The maximum binding capacity, however, as determined by the values of FGFR- 1 bound at saturating ligand concentrations, was about twofold higher for the CD44vRA isoform. Overall, the kinetic study confirms the results presented in Figure 4a and points to the molecular mechanism whereby the vRA mutation induces a specific increase in HS-mediated FGFR-1 binding and activation.

To analyze the mitogenic activity of cell surface-bound FGF-2, we assessed the ability of fixed Namalwa transfectants incubated with FGF-2 (termed FGF-2-immobilized fixed Namalwa transfectants) to stimulate the proliferation of BaF-32 cells 
expressing FGFR-1 (8). FGF-2-immobilized fixed Namalwa-CD44vRA cells stimulated a proliferative response in cocultured $\mathrm{BaF}-32$ cells that was similar to (Figure $4 c$, inset), or as shown in different experiments, lower than (Figure 4, $c$ and d) that of the BaF-32 cells incubated with FGF-2 and heparin (positive control). In all three experiments, however, FGF-2-immobilized fixed Namalwa-CD44vRA cells stimulated BaF-32 cells to a greater extent than did the corresponding Namalwa-CD44v3-v10 cells. We further show (Figure 4d) that anti-CD44v3 mAb reduced the proliferative response of $\mathrm{BaF}-32$ cells stimulated with FGF-2-immobilized fixed NamalwaCD44vRA cells (bars 6,7 , and 8 ) or with the corresponding Namalwa-CD44v3-v10 cells (bars 3, 4, and $5)$. BaF-32 cells incubated with FGF-2 alone or heparin alone showed only a background level of cell proliferation, similar to the proliferation rate of BaF-32 cells stimulated with FGF-2-immobilized fixed Namalwa-CD44s or Namalwa-Neo cells (Figure $4 \mathrm{c}$ and inset). We also demonstrate (Figure $4 \mathrm{~d}$; bars
13, 14, and 15) that fixed RA synovial fluid cells, endogenously loaded with FGF-2 (see Figure 5b), can also stimulate the proliferation of BaF-32 cells. This proliferation was reduced following the addition of anti-CD44v3 mAb (not shown).

Enhanced binding of FGFR-1 to cells isolated from synovial fluid of RA patients. Based on the enhanced binding of FGFR-1 to Namalwa cells expressing CD44vRA, it was important to test and confirm these findings in the authentic cells isolated from the synovial fluid of patients with joint diseases. Flow-cytometry analysis of $18 \mathrm{RA}$ patients and $6 \mathrm{OA}$ patients showed that although joint synovial fluid cells of RA and OA patients expressed pan-CD44 proteoglycan to a nearly equal extent, only RA synovial fluid cells expressed CD44v3 and CD44v6 epitopes (a representative data in Figure 5a). Furthermore, immunostaining with anti-FGF-2 Ab to detect the presence of FGF- 2 on the cell surface of synovial fluid cells revealed that RA and OA cells contain similar levels of FGF-2 (Figure 5b, insets). RA synovial fluid cells, however, bound soluble FGFR-1 more
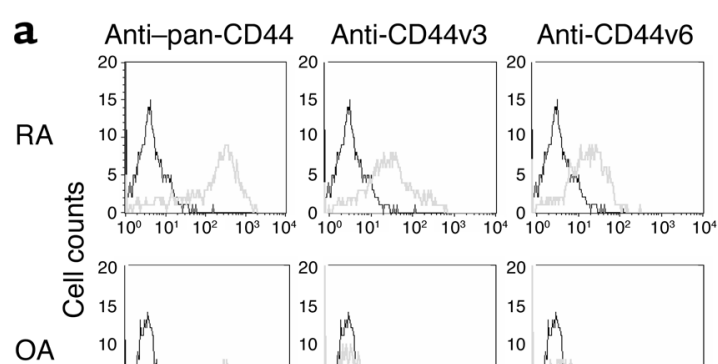

$\mathrm{OA}$
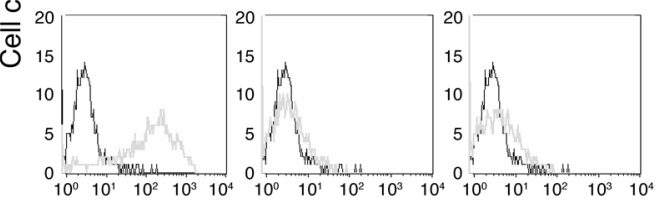

Mean fluorescence intensity

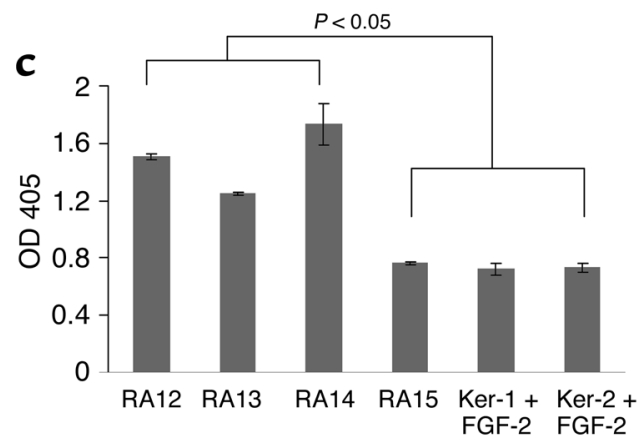

b

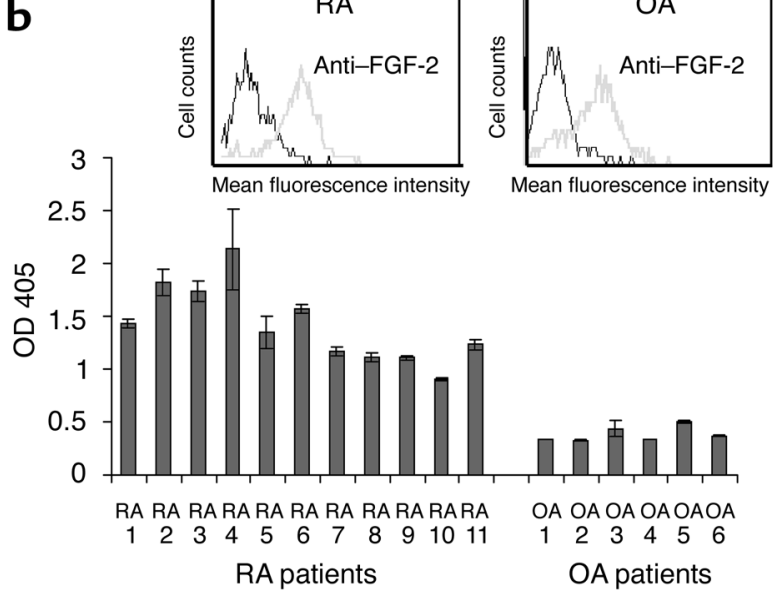

Figure 5

Synovial fluid cells from RA patients bind soluble FGFR-1. (a) CD44 expression on synovial fluid cells from RA and OA patients. Cells collected from the joints of an RA or an OA patient were analyzed by flow cytometry using anti-pan CD44 mAb (F-10-44-2), anti-CD44v3 mAb, or antiCD44v6 mAb. The first histogram in each panel shows staining with second Ab alone. Similar flow-cytometric histograms were recorded in 11 samples of RA patients and 6 of OA patients. (b) Enhanced binding of soluble FGFR-1 to synovial fluid cells of RA patients. Cells from the joints of 11 RA and 6 OA patients were incubated with soluble FGFR-1 conjugated to AP, in the presence (not shown) or absence (b) of FGF-2. The interaction of the FGFR-1 with the FGF-2, bound to the joint cells, was detected using an AP substrate at OD 405. Similar results were observed in the presence of FGF-2 (not shown). Insets: Binding of anti-FGF-2 Ab to joint cells of RA and OA patients to assess the endogenous FGF-2 inclusion in these cells. The first histogram in each inset shows staining with second Ab only (goat anti-rabbit Fab'-FITC). Equal levels of endogenous FGF-2 in RA and OA cells were observed in samples of 11 RA and 6 OA patients. (c) Enhanced binding of soluble FGFR-1 is dependent on CD44vRA expression on RA synovial fluid cells. The binding of FGFR-1 to three patient samples (RA12, RA13, and RA14) of RA synovial fluid cells expressing CD44vRA, one patient sample (RA15) of RA synovial fluid cells expressing CD44v3-v10, and two samples of primary human keratinocytes (Ker-1 and Ker-2) expressing CD44v3-v10 were analyzed as indicated in b. Note that the keratinocytes were loaded with FGF-2 because, unlike synovial fluid cells, they do not contain the endogenous growth factor. Statistical analysis of the principal groups is shown. 

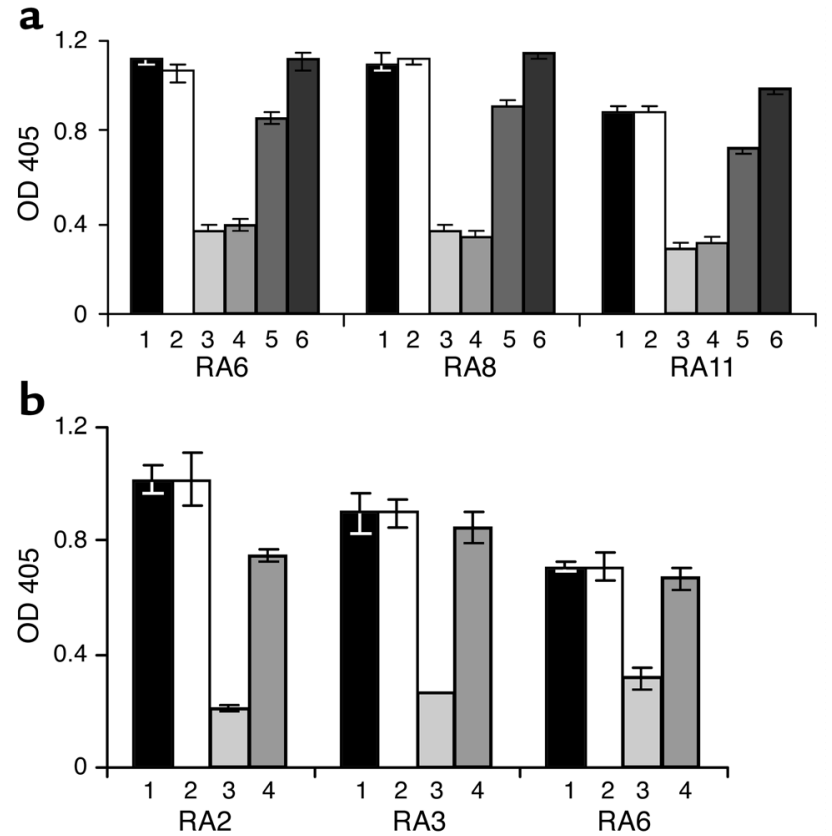

\section{Figure 6}

Synovial fluid cells from RA patients bind soluble FGFR-1 in a CD44vdependent manner. (a) Binding of soluble FGFR-1 to synovial fluid cells of RA patients is CD44v3 associated. Synovial fluid cells from three RA patients (RA6, RA8, RA11) were incubated with soluble FGFR-1 conjugated to AP in the presence of medium (bar 1 ) isotypematched control immunoglobulin (bar 2), or $1 \mu \mathrm{g}$ (bar 3), $300 \mathrm{ng}$ (bar 4), $30 \mathrm{ng}$ (bar 5), and $1 \mathrm{ng}$ (bar 6) anti-CD44v3 mAb. The interaction of FGFR-1 with FGF-2, bound to the joint cells, was analyzed as indicated in Figure $5 \mathrm{~b}$. Anti-CD $44 \mathrm{v} 3 \mathrm{mAb}$ reduced the binding of soluble FGFR-1 to the synovial fluid cells in a dose-dependent manner. The highest concentration ( $1 \mu \mathrm{g}$ ) of anti-CD44v3 mAb reduced FGFR-1 binding to synovial fluid cells from additional four RA patients (not shown). (b) Anti-CD44v3 mAb, but not anti-panCD44mAb (F-10-44-2), directed against a constant epitope inhibits the binding of soluble FGFR-1 to synovial fluid cells of RA patients. Synovial fluid cells from three RA patients (RA2, RA3, RA6) were incubated with soluble FGFR-1 conjugated to AP in the presence of medium (bar 1), isotype matched control immunoglobulin (bar 2), $1 \mu \mathrm{g}$ anti-CD44v3 mAb (bar 3), and $1 \mu \mathrm{g}$ anti-pan CD44mAb (bar 4). The interaction of the FGFR-1 with FGF-2 bound to the joint cells, was analyzed as described in Figure 5 b.

intensively than did OA synovial fluid cells, regardless whether FGF-2 was added to the cells (not shown) or not (Figure $5 \mathrm{~b}$ ). Addition of heparin at saturated concentrations ( 2 and $5 \mu \mathrm{g} / \mathrm{ml}$ ) to OA cells did not enhance the binding of FGFR-1 to these cells (not shown). Moreover, RA synovial fluid cells expressing the CD44vRA variant (detected by PstI digestion) bound soluble FGFR-1, as indicated by analyzing CD44vRA-positive samples from three RA patients. In contrast, RA synovial fluid cells and FGF-2 externally loaded primary keratinocytes expressing the wild-type CD44v3-v10 (our finding and ref. 28), bound considerably less of this receptor, as indicated by analyzing CD44v3-v10-positive samples from one RA patient and two keratinocyte donors (Figure 5c). The binding of soluble FGFR-1 to synovial fluid cells of RA patients was inhibited in a dose-dependent manner by anti-CD44v3 mAb (Figure 6a) but not by F-10-44-2 anti-pan CD $44 \mathrm{mAb}$ (Figure $6 \mathrm{~b})$, indicating that FGFR-1 binding is targeted to the v3 exon of cell surface CD44.

\section{Discussion}

A novel CD44 variant CD44vRA was found in the synovial fluid cells from 23 out of 30 examined RA patients. When expressed in a model of Namalwa cells or in original synovial fluid cells of RA patients, it conferred an enhanced capacity to bind soluble FGFR-1 via FGF-2 immobilized on the cell surface CD44 isoform and to stimulate the proliferation of BaF-32 cells bearing FGFR-1. Because FGF-2 was shown to play an active role in the inflammatory response and in diseaseassociated angiogenesis (see below), we suggest that enhanced binding and activation of FGFR-1 by CD44vRA-expressing synovial fluid cells of RA patients play a role in the RA pathology.

The illegitimate transcription of the intronic CAG flanking exon v5 of CD44vRA is presumably a consequence of misregulation of the splicing machinery in this molecule. Unknown genetic or environmental factors or a combination of both may modify the relative abundance, tissue distribution, or activity of serine-arginine, or of heterogeneous nuclear ribonucleoprotein splicing factors that antagonistically control the differential splicing (31). The CAG-containing splicing junction may be particularly susceptible to such changes, resulting in the CAG inclusion in the CD44 mRNA sequence. A similar mechanism may influence the CD44 splicing machinery of other autoimmune diseases. In this context, it should be mentioned that mutations located in noncoding regions such as those affecting $5^{\prime}$ and $3^{\prime}$ splice sites, branch sites, or polyadenylation signals, are frequently $(\sim 15 \%)$ the cause of genetic diseases (32).

For efficient autocrine or paracrine presentation to the relevant neighboring receptors, FGF-2, like other heparin-binding growth factors, must be assembled on cell surface HS proteoglycans $(13,33)$. The proteoglycan nature of CD44 of cell surfaces is well established $(8,9$, 30). CD44 includes seven potential consensus single serine-glycine (SG) or double SGSG assembly sites for glycosaminoglycans (GAGs), such as HS or chondroitin sulfate. It was found, however, that the assembly of HS is determined by eight amino acids located downstream from the exon v3 SGSG motif. Because exon v3 of the CD44 proteoglycan is the only one containing this sequence in the context of the SG or SGSG motif, HS assembly is confined to this exon (30). The sequestering of HS-binding growth factors on the v3 exon of CD44 proteoglycan has been well documented (5-12). Moreover, Sherman and colleagues (12) demonstrated that the addition of anti-CD $44 \mathrm{v} 3 \mathrm{mAb}$ to limb bud mesenchymal cells cocultured in the presence of FGF-8, with UV-irradiated Namalwa cells expressing CD44v3-v10, markedly reduced the proliferation of the FGFR-bearing mesenchymal cells. Similarly, we show here that 
anti-CD44v3 mAb specifically reduces the binding of soluble FGFR-1 to synovial fluid cells expressing the RA variant of CD44 (Figure 6) and the proliferation of BaF-32 cells after stimulation with Namalwa CD $44 \mathrm{v}$ transfectants (Figure 4d) or RA synoviocytes (not shown). Cumulatively, these findings unequivocally show that the HS-binding growth factor is attached to the v3 exon. HS attached to the v3 of CD44 proteoglycan, which can potentiate the binding of growth factors, was detected on, among other cells, inflamed synovial membrane macrophages, and such expression is greatest in cells immobilizing high levels of FGF-2 (8).

The trinucleotide (CAG) insertion between exon v4 and exon $\mathrm{v} 5$, interpolating an alanine residue at position 346 , constitutes the only difference between CD44vRA and CD44v3-v10 transcripts (Figure 1). Interestingly, inclusion of this single amino acid is sufficient to confer upon FGF-immobilized CD44vRA the capacity for enhanced binding of soluble FGFR-1 and augmented mitogenic activity, without any significant change in the overall FGF-2 binding ability of CD44associated HS chains. These findings imply that, although quantitatively CD44vRA and CD44v3-v10 display a similar HS-dependent FGF-2 binding capacity, qualitatively, FGF-2-immobilized CD44vRA more effectively promotes interaction with FGFR-1. Two alternative mechanisms, not necessarily mutually exclusive, may account for this observation. It is conceivable that the local structural change induced by alanine insertion may indirectly affect the structure of the more distally v 3 exon-attached HS chain so that it can now act as a superior growth factor activator. This, in turn, could result from a local effect on the attachment or recruitment of HS-modifying enzymes responsible primarily for the HS sulfation pattern, including charge density and distribution, critical to its activating or inhibitory functions. Both activities have been implicated in the control of growth factor function in vitro (34) and in vivo (35).

Alternatively, or in addition, the change in the primary sequence of the CD44vRA protein could modify the orientation of existing protein-associated HS structures, enabling them to bind FGF-2 and its receptor in a superior functional form. Analysis of the binding kinetics strongly implies the presence of additional effective and specific binding sites for FGFR-1 on CD44vRA, compared with CD44v3-v10, rather than a change in affinity, as responsible for the new steady-state level obtained. The availability of additional binding sites for FGFR-1 on CD44vRAassociated HS could result from exposure of an existing site that is masked in the CD44v3-v10 isoform by local conformational changes in the core protein or the creation of new binding sites with similar affinity, specific to the CD44vRA-associated HS. The conformational change related to the modified sequence of CD44vRA is demonstrated, using a mAb that was found to bind at higher affinity to Namalwa cells expressing CD44vRA than to Namalwa cells expressing the wild-type CD44v3-v10, although the two transfectants expressed the same level of CD44 (our unpublished observations).

FGF-2, generated at least partly by the joint synoviocytes of RA patients (36), is tightly associated with the exacerbation of RA pathology. Elevated levels of FGF-2 were detected in the synovial tissue (36), synovial fluid (37), and serum (38) of RA patients. FGFR 1, a highaffinity receptor for FGF-2, is also expressed in a variety of RA synovial cells, including endothelial cells (39) (reviewed in ref. 40). The interaction of this growth factor with its cell surface receptor induces, directly or indirectly, osteoclastogenesis and osteocartilaginous destruction in the joints of RA patients (41), as well as in those of rats with adjuvant-induced arthritis (AIA) (37). It was further shown (37) that in rats with AIA, FGF-2 induces, either directly or via stimulation of VEGF release (42), the formation of a new vascular network that supports the inflammatory process.

Although OA synovial fluid cells lack the v3 exon product, they immobilize FGF-2 to nearly the same extent as RA cells (Figure $5 \mathrm{~b}$, insets). This finding suggests that OA synovial fluid cells bind FGF-2 via HS of non-CD44 proteoglycan origin. Nevertheless, FGF-2 bound on OA cells is less potent than this growth factor bound on RA cells, because only RA cells show enhanced binding of FGFR-1 (Figure 5b). We can assume, therefore, that binding of FGF-2 to the v3-attached HS of CD44 proteoglycan (expressed on RA, but not on OA cells) markedly improves the potency of this growth factor.

In conclusion, we have identified, we believe for the first time, a novel variant of CD44 in the synovial fluid cells of RA patients. This exclusive CD44vRA variant interacts with FGF-2 via v3-attached HS in a way that allows enhanced binding and activation of FGFR-1, an event that may contribute to the RA inflammatory process. The exclusive structure of synoviocyte CD44vRA should also allow selective targeting, e.g., with $\mathrm{mAb}$, that could interfere with the vicious inflammatory cascade, leading to joint destruction.

\section{Acknowledgments}

This work was supported by the Society of Research Associates of the Lautenberg Center (New York, New York, USA) and by a grant from ProChon Biotech Ltd. We thank Israel Vlodavsky for the BaF-32 cells, Alexandra Mahler for her editorial assistance, and Sharon Saunders for typing the manuscript.

\footnotetext{
1. Naor, D., Nedvetzki, S., Golan, I., Melnik, L., and Faitelson, Y. 2002. CD44 in cancer. Crit. Rev. Clin. Lab. Sci. 39:527-579.

2. Naor, D., Vogt Sionov, R., and Ish-Shalom, D. 1997. CD44: structure, function, and association with malignant process. Adv. Cancer Res. 71:241-319.

3. Lesley, J., Hyman, R., and Kincade, P.W. 1993. CD44 and its interaction with extracellular matrix. Adv. Immunol. 54:271-335.

4. Van Weering, D.H.J., Baas, P.D., and Bos, J.L. 1993. A PCR-based method for the analysis of human CD44 splice products. PCR Methods Appl. 3:100-106.

5. van der Voort, R., et al. 1999. Heparan sulfate-modified CD44 promotes hepatocyte growth factor/scatter factor-induced signal transduction through the receptor tyrosine kinase c-Met. J. Biol. Chem. 274:6499-6506.
} 
6. Wielenga, V.J.M., et al. 2000. Expression of c-Met and heparan-sulfate proteoglycan forms of CD44 in colorectal cancer. Am. J. Pathol. 157:1563-1573.

7. van der Voort, R., Keehnen, R.M.J., Beuling, E.A., Spaargaren, M., and Pals, S.P. 2000. Regulation of cytokine signaling by B cell antigen receptor and CD40-controlled expression of heparan sulfate proteoglycans. J. Exp. Med. 192:1115-1124.

8. Jones, M., Tussey, L., Athanasou, N., and Jackson, D.G. 2000. Heparan sulfate proteoglycan isoforms of the CD44 hyaluronan receptor induced in human inflammatory macrophages can function as paracrine regulators of fibroblast growth factor action. J. Biol. Chem. 275:7964-7974.

9. Bennett, K.L., et al. 1995. CD44 isoforms containing exon v3 are responsible for the presentation of heparin-binding growth factor. J. Cell. Biol. 128:687-698.

10. Yu, W.-H., Woessner, J.F., Jr., McNeish, J.D., and Stamenkovic, I. 2002. CD44 anchors the assembly of matrilysin/MMP-7 with heparin-binding epidermal growth factor precursor and ErbB4 and regulates female reproductive organ remodeling. Genes Dev. 16:307-323.

11. Grimme, H.U., et al. 1999. Colocalization of basic fibroblast growth factor and CD44 isoforms containing the variably spliced exon v3 (CD44v3) in normal skin and in epidermal skin cancers. Br. J. Dermatol. 141:824-832.

12. Sherman, L., Wainwright, D., Ponta, H., and Herrlich, P. 1998. A splice variant of CD44 expressed in the apical ectodermal ridge presents fibroblast growth factors to limb mesenchyme and is required for limb outgrowth. Genes Dev. 12:1058-1071.

13. Yayon, A., Klagsbrun, M., Esko, J.D., Leder, P., and Ornitz, D.M. 1991. Cell surface, heparin-like molecules are required for binding of basic fibroblast growth factor to its high affinity receptor. Cell. 64:841-848.

14. Rapraeger, A.C., Krufka, A., and Olwin, B.B. 1991. Requirement of heparan sulfate for bFGF-mediated fibroblast growth and myoblast differentiation. Science. 252:1705-1708.

15. Ornitz, D.M., et al. 1992. Heparin is required for cell-free binding of basic fibroblast growth factor to a soluble receptor and for mitogenesis in whole cells. Mol. Cell Biol. 12:240-247.

16. Bourguignon, L.Y.W. 2001. CD44-mediated oncogenic signaling and cytoskeleton activation during mammary tumor progression. J. Mammary Gland Biol. Neoplasia. 6:287-297.

17. Zahalka, M.A., Okon, E., Gosslar, U., Holzmann, B., and Naor, D. 1995 Lymph node (but not spleen) invasion by murine lymphoma is both CD44- and hyaluronate-dependent. J. Immunol. 154:5345-5355.

18. Nedvetzki, S., et al. 1999. CD44 involvement in experimental collageninduced arthritis (CIA). J. Autoimmun. 13:39-47.

19. Weiss, L., et al. 2000. Induction of resistance to diabetes in non-obese diabetic mice by targeting CD44 with a specific monoclonal antibody. Proc. Natl. Acad. Sci. U. S. A. 97:285-290.

20. Mikecz, K., Brennan, F.R., Kim, J.H., and Glant, T.T. 1995. Anti-CD44 treatment abrogates tissue oedema and leukocyte infiltration in murine arthritis. Nat. Med. 1:558-563.

21. Verdrengh, M., Holmdahl, R., and Tarkowski, A. 1995. Administration of antibodies to hyaluronanreceptor (CD44) delays the start and ameliorates the severity of collagen II arthritis. Scand. J. Immunol. 42:353-358.

22. Witting, B., Schwärzler, C., Föhr, N., Günthert, U., and Zöller, M. 1998. Cutting edge: curative treatment of an experimentally induced colitis by a CD44 variant V7-specific antibody. J. Immunol. 161:1069-1073.

23. Brocke, S., Piercy, C., Steinman, L., Weissman, I.L., and Veromaa, T. 1999. Antibodies to CD44 and integrin $\alpha_{4}$, but not L-selectin, prevent central nervous system inflammation and experimental encephalomyelitis by blocking secondary leukocyte recruitment. Proc. Natl. Acad. Sci. U. S. A 96:5896-6901.
24. Wallach-Dayan, S.B., et al. 2001. CD44-dependant lymphoma cell dissemination: a cell surface CD44 variant, rather than standard CD44, supports in vitro lymphoma cell rolling on hyaluronic acid substrate and its in vivo accumulation in the peripheral lymph nodes. J. Cell. Sci. 114:3463-3477.

25. Zhang, Z., Coomans, C., and David, G. 2001. Membrane heparan sulfate proteoglycan-supported FGF2-FGFR1 signaling. J. Biol. Chem. 276:41921-41929.

26. Richard, C., Liuzzo, J.P., and Moscatelli, D. 1995. Fibroblast growth factor- 2 can mediate cell attachment by linking receptors and heparan sulfate proteoglycans on neighboring cells. J. Biol. Chem. 270:24188-24196.

27. Filla, M.S., Dam, P., and Rapraegar, A.C. 1998. The cell surface proteoglycan syndecan-1 mediates fibroblast growth factor- 2 binding and activity. J. Cell Physiol. 174:310-321.

28. Hoffmann, M., et al. 1991. CD44 splice variants confer metastatic behavior in rats: homologous sequences are expressed in human tumor cell lines. Cancer Res. 51:5292-5297.

29. Screaton, G.R., et al. 1992. Genomic structure of DNA encoding the lymphocyte homing receptor CD44 reveals at least 12 alternatively spliced exons. Proc. Natl. Acad. Sci. U. S. A. 89:12160-12164.

30. Greenfield, B., et al. 1999. Characterization of the heparan sulfate and chondroitin sulfate assembly sites in CD44. J. Biol. Chem. 274:2511-2517.

31. Cáceres, J.R., and Kornbliht, A.R. 2002. Alternative splicing: multiple control mechanisms and involvement in human diseases. Trends Genet. 18:186-193.

32. Krawczak, M., Reiss, J., and Cooper, D.N. 1992. The mutational spectrum of single base-pair substitutions in mRNA splice junctions of human genes: causes and consequences. Hum. Genet. 90:41-54.

33. Ornitz, D.M. 2000. FGFs, heparan sulfate and FGFRs: complex interactions essential for development. Bioessays. 22:108-112.

34. Aviezer, D., et al. 1994. Differential structural requirements of heparin and heparan sulfate proteoglycans that promote binding of basic fibroblast growth factor to its receptor. J. Biol. Chem. 269:114-121.

35. Arikawa-Hirasawa, E., Watanabe, H., Takami, H., Hassell, J.R., and Yamada, Y. 1999. Perlecan is essential for cartilage and cephalic development. Nat. Genet. 23:354-358.

36. Qu, Z., Huang, X.-N., Ahmadi, P., Andresevic, J., Planck, S.R., Hart, C.E, and Rosenbaum, J.T. 1995. Expression of basic fibroblast growth factor in synovial tissue from patients with rheumatoid arthritis and degenerative joint disease. Lab. Invest. 73:339-346.

37. Yamashita, A., et al. 2002. Fibroblast growth factor-2 determines severity of joint disease in adjuvant-induced arthritis in rats. J. Immunol. 168:450-457.

38. Nagashima, M., et al. 2000. Effects of combinations of anti-rheumatic drugs on the production of vascular endothelial growth factor and basic fibroblast growth factor in cultured synoviocytes and patients with rheumatoid arthritis. Rheumatology. 39:1255-1262.

39. Byrd, V., Zhao, X.-M., McKeehan, W.L., Miller, G.G., and Thomas, J.W. 1996. Expression and functional expansion of fibroblast growth factor receptor $\mathrm{T}$ cells in rheumatoid synovium and peripheral blood of patients with rheumatoid arthritis. Arthritis Rheum. 39:914-922.

40. Koch, A.E. 1998. Angiogenesis. Implications for rheumatoid arthritis. Arthritis Rheum. 41:951-962.

41. Manabe, N., et al. 1999. Involvement of fibroblast growth factor-2 in joint destruction of rheumatoid arthritis patients. Rheumatology. 38:714-720.

42. Seghezzi, G., et al. 1998. Fibroblast growth factor-2 (FGF-2) induces vascular endothelial growth factor (VEGF) expression in the endothelial cells of forming capillaries: an autocrine mechanism contributing to angiogenesis. J. Cell Biol. 141:1659-1673. 